\title{
AnnexinA7 and CAP1 are associated with regulating tumor cell adhesion molecule expression and biological behavior
}

\section{Type}

Research paper

\section{Keywords}

hepatocellular carcinoma, adhesion molecule, AnnexinA7, CAP1, Biological behaviors

\begin{abstract}
Introduction

To find out the correlations between the effects of down-regulating AnnexinA7 and CAP1 gene on cell adhesion factors and the biological behavior of Hca-p cells cells, and finally infer the relationship between the two genes.

Material and methods

Western blot ,qRT-PCR,immunocytochemistry, CCK8 cell proliferation, flow cytometry, lymph node adhesion and transwell chamber assay testing .

Results

AnnexinA7 and CAP1 were consistent with the regulation of the expression of the adhesion molecules such as FAK, Src, Paxillin and E-cadherin. At the mRNA and protein levels, the expression of FAK, Src and Paxillin were increased with down-regulated AnnexinA7 and CAP1 genes, while E-cadherin was down-regulated in the change of these two genes. And the low expression of AnnexinA7 could affect the expression of CAP1 in mRNA and protein levels. otherwise, the localization of AnnexinA7 and CAP1 in hepatocellular carcinoma cells was also the same. After down-regulating the expression of CAP1, the functions of proliferation, lymph node adhesion and invasion were increased and early apoptotic ability was decreased in Hca-P cells.
\end{abstract}

\section{Conclusions}

AnnexinA7 and CAP1 could control the expression of these adhesion molecules in the same trend. We speculated they may be co-localization. And AnnexinA7 gene may be related to the molecular mechanism of CAP1 gene, which is likely to have a consistent effect on cell adhesion molecules and be closely related to the biological behaviors of Hca-P cells.AnnexinA7 and CAP1 may play an inhibitory role in lymph node metastasis to provide a reliable basis for the early identification of lymphatic metastasis in hepatocellular carcinoma. 


\title{
AnnexinA7 and CAP1 are associated with regulating tumor cell adhesion molecule expression and biological behavior
}

\author{
Li Wanga, Yang Wang ${ }^{\mathrm{c}}$, Zhiyan Liu ${ }^{\mathrm{a}}$,Ying Yang ${ }^{\mathrm{a}}$, Yixia Zhou ${ }^{\mathrm{a}}$, Jun Zhang ${ }^{\mathrm{b}}$ and \\ Jianwu Tang ${ }^{\mathrm{b}, *}$
}

${ }^{a}$ Guizhou Medical University, Guiyang 550004, China

${ }^{\mathrm{b}}$ Dalian Medical University, Dalian 116044, Liaoning, China.

${ }^{\mathrm{c}}$ Guizhou Medical University Affiliated Hospital, Guiyang ,550001, China

*Correspondence author: Jianwu Tang, Department of Pathology, Dalian Medical University, 9- western section, Lvshun south street, Dalian 116044, Liaoning, China. E-mail: jianwutang1@163.com

\begin{abstract}
BACKGROUND: Hca-p and Hca-F were chosen and established from the accumulation of multiple genetic alterations and complicated molecular pathways by our group in previous studies as a pair of syngeneic mouse hepatocarcinoma ascites cell lines. The two cell lines showed different lymph node metastasis potential (Hca-p $<30 \%$, Hca-F $>70 \%$ ) when inoculated subcutaneously in Chinese 615 mice. The suppressive subtracted hybridization technique, gene chip and comparative proteomics were applied to screen out differentially expressed genes and proteins between the two cell lines including AnnexinA7 and CAP1.AnnexinA7 was found to impact on cell growth, differentiation, secretion, invasion, migration, proliferation and apoptosis ability of tumor, as one of the key potential genes of deciding liver cancer lymphatic metastasis identified by our group anteriorly. CAP1 is an important cytoskeletal regulatory protein which is related with proliferation, migration, invasion and apoptosis and other biological behaviors in tumor cells. However, the research on the relationship between AnnexinA7 and cytoskeletal proteins is scarce, such as adenylate cyclase-associated protein 1 (CAP1).
\end{abstract}

OBJECTIVE: To find out the correlations between the effects of down-regulating AnnexinA7 and CAP1 gene on cell adhesion factors and the 
biological behavior of Hca-p cells cells, and finally infer the relationship between the two genes.

METHODS: AnnexinA7 and CAP1 were down-regulated by shRNA interference in Hca - P cells. Western blot and qRT-PCR were used to examine the regulation of AnnexinA7 and CAP1 on the expression of cell adhesion-related molecules, and then immunocytochemistry was applied to determine the further relationship between these two genes. CCK8 cell proliferation, flow cytometry, lymph node adhesion and transwell chamber assay testing were performed to detect the proliferation, apoptosis, adhesion and invasion ability of Hca - P cells under interference effect of CAP1 gene.

RESULTS: We found that AnnexinA7 and CAP1 were consistent with the regulation of the expression of the adhesion molecules such as FAK, Src, Paxillin and E-cadherin. At the mRNA and protein levels, the expression of FAK, Src and Paxillin were increased with down-regulated AnnexinA7 and CAP1 genes, while E-cadherin was down-regulated in the change of these two genes. And the low expression of AnnexinA7 could affect the expression of CAP1 in mRNA and protein levels. otherwise, the localization of AnnexinA7 and CAP1 in hepatocellular carcinoma cells was also the same. After down-regulating the expression of CAP1, the functions of proliferation, lymph node adhesion and invasion were increased and early apoptotic ability was decreased in Hca-P cells.

CONCLUSIONS: AnnexinA7 and CAP1 could control the expression of these adhesion molecules in the same trend. We speculated they may be co-localization. And AnnexinA7 gene may be related to the molecular mechanism of CAP1 gene, which is likely to have a consistent effect on cell adhesion molecules and be closely related to the biological behaviors of Hca-P cells. On the basis of the above, we indicated that AnnexinA7 and CAP1 may play an inhibitory role in lymph node metastasis to provide a reliable basis for the early identification of lymphatic metastasis in hepatocellular carcinoma.

Keywords: AnnexinA7; CAP1; Hepatocellular carcinoma; Adhesion molecule; Biological behaviors 


\section{Introduction}

Hepatocellular carcinoma ( $\mathrm{HCC}$ ) is still the high fatality rate of malignant tumor of epithelial origin. As the earliest transfer way of malignant tumors, lymphatic metastasis is one of the main reasons for high recurrence and prognosis caused by primary cancer invasion and migration. However, the detailed mechanism of transfer channels is still not completely known.

AnnexinA7(ANXA7) is identified as one of the key potential genes of deciding liver cancer lymphatic metastasis by our preliminary work, which impacts on cell growth, differentiation, secretion, invasion, migration, proliferation and apoptosis ability of a various tumor. Moreover, as a widely distributed member of the $\mathrm{Ca} /$ Phospholipid-binding annexin family, AnnexinA7 is active with $\mathrm{ca}^{2}+$ dependency membrane fusion, could promote the membrane aggregation, fusion, adhesion, transportation, and activate the GTP enzyme production with mediated $\mathrm{ca}^{2+} /$ GTP signal transduction pathways. Other studies have found that the defects of cytoskeleton proteins were related to affect the expression of AnnexinA7 on the human red cell membrane protein. Nevertheless, the specific relationship between AnnexinA7with the cytoskeleton proteins is still unclear [1-4].

Adenylate cyclase-associated protein1 ( CAP1) is a kind of conservative cytoskeleton regulatory protein which participated in regulation of actin and Ras/cAMP signaling pathways, and its expression change regulates numerous important cellular processes, such as cell adhesion, proliferation, migration, invasiveness ,apoptosis and so on[5,6].CAP1 plays distinct roles in different types of tumor cells. For instance, some studies have shown that lack of CAP1 leads to increasing cell volume and significantly promotes the agglomeration of actin layer board in HeLa cells, with enhancing cell migration and proliferation by the activation of FAK (Focal Adhesion Kinase)[7].In glioma cancer cells, over-expressed CAP1 protein has been proved to impact on the situation of lymphatic metastasis[8].Down-regulated expression of CAP1 can decrease the cell migration and also be involved in tumor cells lymphatic metastasis in esophageal squamous cancer cells [9].Although the research on relationship between CAP1 and tumor 
metastasis has been gradually deepening in recent years, it seems that the influence of tumor biology activities among different malignant cells is not the same.

Adhesion molecule is an important component of the extracellular matrix, which is not only involved in tumor microenvironment, but also participates in the complex cellular signal transduction. At the same time, it could lead to weaken the homogeneity adhesion strength, and heighten the heterogeneity adhesion strength, then to affect all kinds of cell migration and invasion ultimately [10]. FAK is one of the types of the cytoplasm of body protein tyrosine kinase and expresses in a lot of epithelial tumor cells effecting on tumor growth, proliferation, invasion, metastasis and angiogenesis. Src is one member of the SRC family of tyrosine kinase, and involved in cell adhesion, proliferation, apoptosis, differentiation and movement process. Paxillin is the key bridging protein to spot signaling molecules stick together, which is the focus of the downstream molecules of FAK. The abnormal expression of paxillin is associated with tumor occurrence, proliferation, adhesion, invasion, skeleton restructuring and transfer ability. E-cadherin is closely related with tumor invasion and metastasis of a calcium mucin, which can participate in cell polarity, cell morphology and maintain the integrity of the cell structure.

The relationship between AnnexinA7 and CAP1 was rarely found by retrieving concerned literatures. Therefore, shRNA interference technology was used to transfect Hca-P cells suppressed the expression of AnnexinA7 and CAP1.Western Blot and qRT-PCR were applied to check the influence on the gene and protein expression levels of FAK, Src, Paxillin and E-cadherin by cutting AnnexinA7 and CAP1 gene, including the influence between the two genes themselves. Furthermore , we used the cell function test to check the influence of the biological behaviors such as proliferation, apoptosis, adhesion and invasion in $\mathrm{Hca}-\mathrm{P}$ cell. With the continuous development of the molecular biology and proteomics research, we hope our study on AnnexinA7 and CAP1 with adhesion molecules could be the more powerful support to tumor early diagnosis, treatment and prognosis.

\section{Materials and methods}

\subsection{Cell cultures and transfection}


Hca-P cell, as a suspended cell line, has been proved to be an ideal model established in our laboratory. The cells were routinely cultured in the RPMI - 1640 medium(Gibco,USA) containing of $15 \%$ fetal bovine serum (FBS;PAA,USA) in a humidified incubator with $5 \% \mathrm{CO}_{2}$ saturated at $37^{\circ} \mathrm{C}$. The Hca-P Cells being counted $\left(4 \times 10^{5} / \mathrm{ml}\right)$ were seeded into a 24 -well plate $(500 \mu \mathrm{l} /$ well $)$, and $100 \mu 1$ transfection solution(Lipo2000 reagent; Opti-MEM medium) containing shRNA-AnnexinA7, AnnexinA7-NC-unrelated,shRNA-CAP1, CAP1-NC-empty vector were added to culture the experimental cell. After 48 hours, the cells were screened in $20 \%$ FBS media with $400 \mu \mathrm{g} / \mathrm{ml} \mathrm{G} 418$ drug (Invitrogen, USA) to ten days. Through the above procedures, the stable Annexin A7 downregulated (ShRNA-A7) cells, Annexin A7unrelated sequence (shNC-A7) cells, CAP1 downregulated (ShRNA-CAP1) cells and CAP1- empty vector (shNC-CAP1) cells were established.

\subsection{Real-time PCR analysis}

For qRT-PCR analysis the mRNA expression of AnnexinA7, CAP1,FAK,src,Paxillin and E-cadherin ,total RNA was isolated from cells with Trizol reagent(Invitrogen, USA).Reverse transcription of purified RNA was performed using the PrimeScript1 RT reagent kit(Jakara, Japan).Quantification of gene transcripts was performed using the iCycler iQ Multi-Color Real-time PCR Detection System and the iQ SYBR Green Supermix (Bio-Rad), and GAPDH was used as the internal control gene for normalization. Primer Sequences (Takara, Japan) for Annexin A7, CAP1,FAK,src,Paxillin, E-cadherin and GAPDH are listed in Table 1.MXP software and $\Delta \Delta \mathrm{Ct}$ method were used to analyze the results. Differences in mRNA expression level was calculated and displayed as $2(-\Delta \Delta \mathrm{Ct})$.

Table 1 Primer Sequences for AnnexinA7, CAP1,FAK,src,Paxillin, E-cadherin and GAPDH

Gene $\quad$ Forward primer $\left(5^{\prime} \rightarrow 3^{\prime}\right) \quad$ Reverse $\left(3^{\prime} \rightarrow 5^{\prime}\right)$
name

Annexin CCCTGTTCATGCCTCCTACA CACACGCTCTTGAGTTCCTG A7 


\begin{tabular}{|c|c|c|}
\hline \multirow[t]{2}{*}{ CAP1 } & AACCAGGAGAATGTTTCCAA & AAACACCAGGCCAAGCTTCTT \\
\hline & $\mathrm{CCT}$ & \\
\hline \multirow{2}{*}{ FAK } & CCTGGTTATCCTAGCCCGAG & AAGAGATGCCTGACCTTGGTA \\
\hline & AT & AAT \\
\hline \multirow[t]{2}{*}{ src } & TCTCTGTATCCGACTTCGAC & CATCAGCATGTTTGGAGTAGT \\
\hline & AATG & AAGC \\
\hline \multirow[t]{2}{*}{ Paxillin } & GGCAAAGCGTACTGTCGTAA & GTTGACGAAGGGTGTGAAGCA \\
\hline & AGA & \\
\hline E-cadheri & CAGGTCTCCTCATGGCTTTG & CTTCCGAAAAGAAGGCTGTCC \\
\hline $\mathrm{n}$ & $\mathrm{C}$ & \\
\hline \multirow[t]{2}{*}{ GAPDH } & AAATGGTGAAGGTCGGTGTG & CAACAАTCTCCACTTTGCCACT \\
\hline & $\mathrm{AAC}$ & $\mathrm{G}$ \\
\hline
\end{tabular}

\subsection{Protein extraction and Western blot analysis}

Each group of (ShRNA-A7, shNC-A7, ShRNA-CAP1, shNC-CAP1 and Hca-P) cells were collected centrifugally and total protein was extracted with the application of RIPA cracking liquid (Sigma). The concentration of protein was determined by BCA protein kit (Thermo Fisher Scientific, USA). Total protein products wrer dissolved in $6 \times$ loading buffer and boiled at $100{ }^{\circ} \mathrm{C}$ water for 4 minutes. Total protein (50ug) was separated by $12 \%$ or $10 \%$ sodium dodecyl sulfate-polyacrylamide gel (SDS- PAGE). Gels were transferred onto polyvinylidene fluoride (PVDF) membranes (Millipore, USA). Membranes were treated with 5\% non-fat milk in $1 \times$ phosphate-buffered saline-Tween (PBST) solution for $2 \mathrm{~h}$ and then incubated with antibodies against AnnexinA7(1:2000), CAP1(1:1000), FAK (1:1000), Src (1:1000), Paxillin (1:500), E-cadherin (1:500), and GAPDH (1:1500) overnight at $4{ }^{\circ} \mathrm{C}$ followed by incubation with fluorescent secondary antibodies(1:16000)for $1.5 \mathrm{~h}$ at room temperature. Western blot results were visualized by the Odyssey Infrared Imaging System (LI-COR Biosciences, USA). All experiments were conducted three times in 
triplicate. Interest protein expression was represented as a value relative to GAPDH expression.

\subsection{CCK-8 proliferation assay}

The three groups of cells (Hca-P, ShRNA- CAP1, ShNC-CAP1) were collected and counted to the density of $1.5 \times 10^{4} / \mathrm{ml}$. Then the cells were seeded into 96- well plate $(100 \mu \mathrm{l} /$ well $)$ and incubated for $0 \sim 72 \mathrm{~h}$ at $37^{\circ} \mathrm{C}$ incubator in $5 \% \mathrm{CO}_{2}$. CCK-8 solution (Beyotime,10 $\mu$ l/well) was added into the cell suspension regularly. Cell mixture of different time nodes was tested by enzyme standard in $450 \mathrm{~nm}$ absorbance value. Five holes of each experiment group were set up and the results were statistically analyzed by applying repeated data from analysis of variance.

\subsection{Cell apoptosis assay}

Cell apoptosis was analyzed by flow cytometry (BD, USA). After being washing twice with phosphate-buffered saline (PBS), the collected cells were resuspended in $200 \mu \mathrm{l}$ binding buffer. Then $10 \mu \mathrm{l}$ mixture of Annexin V and APC (Vazyme, USA) and $7 \mu 1$ PI solution were added and the cell samples were vortexed and incubated at room temperature for 15 minutes avoiding light. Another $800 \mu$ l Binding buffer was subsequently added. Flow cytometry was performed within one hour.

\subsection{Lymph node adhesion assay}

Lymph nodes of 615 mice were taken from groin, armpit or popliteal fossa to do frozen sections in a sterile environment and dried at room temperature. The three groups of Hca-P cell, ShRNA-CAP1 cell and ShNC-CAP1 cell were counted to $1 \times 10^{6} / \mathrm{ml}$ and dropped on each slice $(200 \mu \mathrm{l} / \mathrm{slice})$. Then these slices were put into cell incubation box for $24 \mathrm{~h}$ and washed three times using precooled PBS liquid after taken out. Methanol was applied to immobilize the experimental samples. Hematoxylin eosin staining (HE staining) was performed and neutral gum was used to seal pieces.

\subsection{Invasion assay}

ECM glue (BD, USA) was melted overnight and mixed with 1640 serum at the ratio of 1: 3. Then $50 \mu 1$ mixture was added to the Transwell chamber (QCM 24-well 
cell invasion assay kit; Sigma) and be placed in a cell incubator for 1h. After the cell density was regulated to $2.5 \times 10^{5} / \mathrm{ml}$, Serum-free single-cell suspension $200 \mu 1$ were added to the upper compartment and the lower chamber was filled with $600 \mu \mathrm{l}$ RPMI-1640 with $15 \%$ FBS at $37{ }^{\circ} \mathrm{C}$ for $24 \mathrm{~h}$. The cells on upper chamber were removed by scraping with a cotton bud. Then the cells on lower chamber were dried at room temperature for $30 \mathrm{~min}$, dyed by $1 \%$ crystal violet solution for $15 \mathrm{~min}$ and counted in five random fields at $40 \times$ magnification with an inverted microscope.

\subsection{Immunofluorescence assay}

Immunofluorescence was used to detect the co-location relation of AnnexinA7 and CAP1.Hca-p cells were collected and washed three times with PBST solution. Nest, $200 \mu 1$ cell suspension was taken in poly-lysine bubble on anti-off slides and dried in the air. The cell slides were fixed with $4 \%$ paraformaldehyde for $30 \mathrm{~min}$ and washed three times on shaker using PBST solution. The cells were punched with $0.1 \%$ Triton X-100 for $10 \mathrm{~min}$ and blocked with 10\% BSA at room temperature for $30 \mathrm{~min}$. After the first antibodies (AnnexinA7 antibody for rat anti-mouse, CAP1 antibody rabbit anti-mouse) were applied, the cells were incubated at $4{ }^{\circ} \mathrm{C}$ overnight. Then, the secondary fluorescent antibody was added avoiding light for 30min. DAPI solution $(5 \mu \mathrm{g} / \mathrm{ml})$ was used to stain cell nucleus for $3 \mathrm{~min}$. The results were imaged using a Laser Scanning Confocal Microscope.

\subsection{Statistical analyses}

Each experiment was repeated 3 times or more, SPSS17.0 software (SPSS company, IN) was used for statistical analysis. All data were expressed as mean \pm SD. Analysis of variance (ANOVA) or one-way ANOVA for repeated measures coupled with the $\mathrm{x}^{2}$-test were used between two independent samples. LSD method was performed for Mean comparison. Spearman grade or chi-square test was used to compare the rate of other related methods, $\mathrm{P}<0.05$ was considered to be statistical significantly.

\section{Results}




\subsection{Transfection effects of ShRNA-A7/ ShNC-A7, ShRNA-CAP1/ ShNC-CAP1 cells}

The Hca-P cells were transfected by PGPUP / GFP / neo-shRNA-Annexin A7, PGPUP / GFP / neo-shNC about Annexin A7, PGPUP / GFP / neo-shRNA-CAP1, and PGPUP / GFP / neo-shNC about CAP1 and observed the expression of the green fluorescent protein with fluorescence microscope. The transfection efficiency of each group was up to $70 \%$ or more (Fig.1 A, B).

A

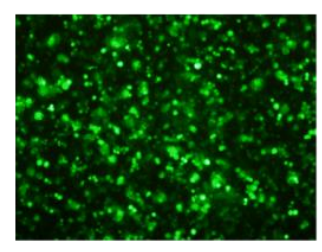

ShRNA- A7

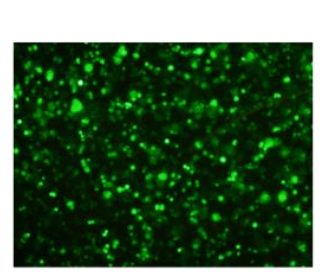

Shic-A7
B
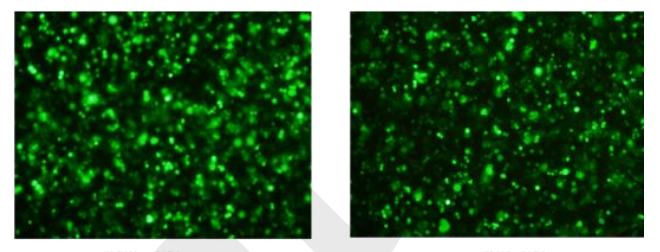

ShNC-CAP1

Fig. 1. (A) (B) The magnification and green fluorescence in stable transfected ShRNA-A7/ ShNC-A7, ShRNA-CAP1/ ShNC-CAP1 cells $(\times 40)$.

\subsection{Downregulation of AnnexinA7 gene affected the expression of adhesion} related molecular in gene and protein levels

At mRNA expression level, AnnexinA7 in ShRNA-A7 group was relatively reduced $82.86 \%$ and $82.78 \%$ than ShNC-A7 group and undisposed Hca-P group respectively $(\mathrm{P}<0.05)$, but there was not obvious difference between ShNC-A7 and Hca-P group ( $\mathrm{P}>0.05) . F A K$ and Src gene expressed in ShRNA-A7 cell group was increased 1.88 times, 1.67 times and 1.78 times ,1.54 times than ShNC-A7 and Hca-P groups respectively, there was no obvious difference between ShNC-A7 and Hca-P group ( $\mathrm{P}>0.05$ ).E-cadherin in ShRNA-A7 group was reduced $49.88 \%$ and $43.01 \%$ than ShNC-A7 and Hca-P groups severally $(\mathrm{P}<0.05)$, no obvious difference between the ShNC-A7 and Hca-P groups (P > 0.05)( Fig.2 A).

On protein expression levels, AnnexinA7 in ShRNA - A7 group was $62.75 \%$ and $60.34 \%$ lower than those in ShNC-A7 and Hca $-\mathrm{P}$ groups $(\mathrm{P}<0.05)$, while it has no difference between the Hca-P and ShNC-A7 groups (P> 0.05). The protein expression of FAK and Src in ShRNA-A7 group was 1.80 and 1.52 times ,or 1.59 and 
1.58 times higher than those in ShNC-A7 and Hca-P groups $(\mathrm{P}<0.05)$, E-cadherin protein in ShRNA - A7 group was 54.27\% and 54.27\% lower than ShNC-A7 and Hca $-\mathrm{P}$ groups $(\mathrm{P}<0.05)$, but these three proteins had no exist significant differences etween the Hca-P and ShNC-A7 groups (P> 0.05) (Fig.2 B).

The results showed that AnnexinA7 could regulate the expression of FAK, Src and E-cadherin in Hca-P cells, and among them FAK and Src were up-regulated with AnnexinA7 declined, but the expression of E-cadherin was suppressed.

A
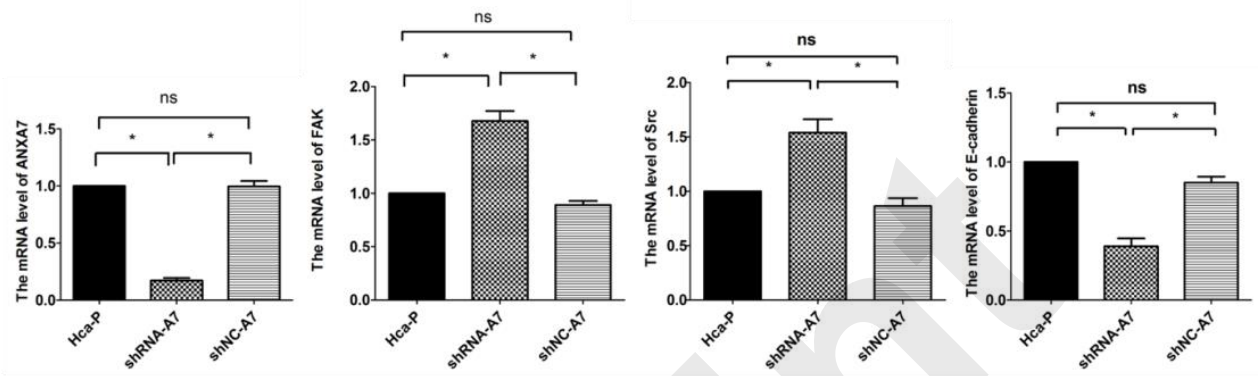

B
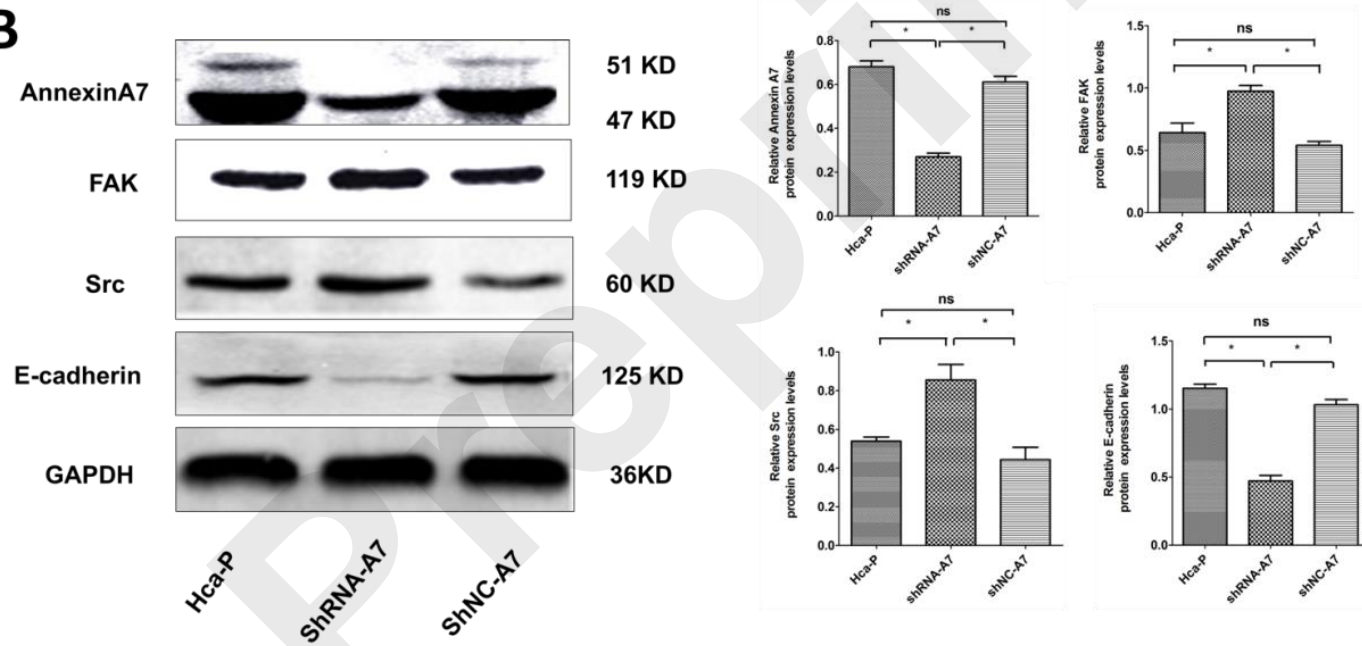

$60 \mathrm{KD}$
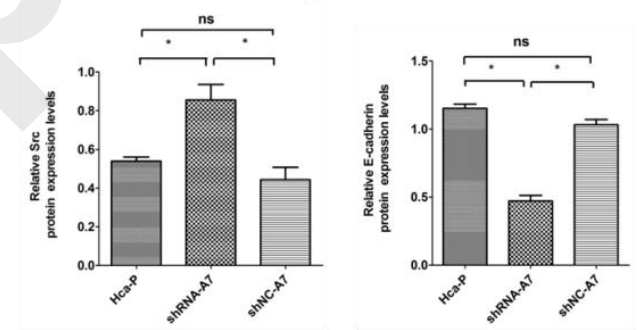

Fig.2. (A)The mRNA expression levels of AnnexinA7, FAK, Src and E-cadherin genes were detected by qRT-PCR. * indicates P $<0.05$, ns represents $\mathrm{P}>0.05$. (B)On the left side of this figure, it shows the protein gel electrophoresis case of these three groups of cells; On the right side of this figure, the protein column comparison was demonstrated of the three groups of cells; $*$ indicates $\mathrm{P}<0.05$, ns represents $\mathrm{P}>0.05$.

\subsection{Downregulation of CAP1 gene affected the expression of adhesion related} molecular in gene and protein levels 
After CAP1 was downregulated, CAP1 in ShRNA - CAP1 group was relatively $60.73 \%$ and $65.25 \%$ (mRNA) lower than ShNC-CAP1 and Hca - P cell groups(P < $0.05)$.

The genes expression of FAK, Src and Paxillin in ShRNA-CAP1 group were increased 2.47 times, 2.18 times and 2.18 times, 1.76 times and 1.67 times, 1.76 times than ShNC-CAP1 and Hca-P groups $(\mathrm{P}<0.05)$.E-cadherin(mRNA) in ShRNA-CAP1 group was decreased by $40.11 \%$ and $43.01 \%$ respectively than ShNC-CAP1 and Hca$\mathrm{P}$ groups $(\mathrm{P}<0.05)$. But there was no obvious difference between the ShNC-CAP1 and Hca-P groups in the expression of the above genes (P > 0.05) (Fig.3 A).

The CAP1 protein expression level in ShRNA-CAP1 group was 50.83\% and $50.29 \%$ lower than ShNC-CAP1 and Hca $-\mathrm{P}$ groups $(\mathrm{P}<0.05)$. The protein expression of FAK, Paxillin and Src in ShRNA-CAP1 group 1.64 times and 1.60 times, 1.67 times and 1.67 times, 1.43 times and 1.43 times higher than the other two groups $(\mathrm{P}<0.05)$.The E-cadherin protein expression level in ShRNA - CAP1 group was decreased $39.6 \%$ and $48.22 \%(\mathrm{P}<0.05)$, but there was no obvious difference between the ShNC-CAP1 and Hca-P groups in the expression of the above protein molecules $(\mathrm{P}>0.05)$. (Fig.3 B)

It was suggested that CAP1 could regulate the expression of FAK, Src, Paxillin and E-cadherin in Hca-P cells. The expressions of FAK, Src and Paxillin were upregulated in CAP1 gene declined cells, but E-cadherin was downregulated with the expression of CAP1 decreased. 
A
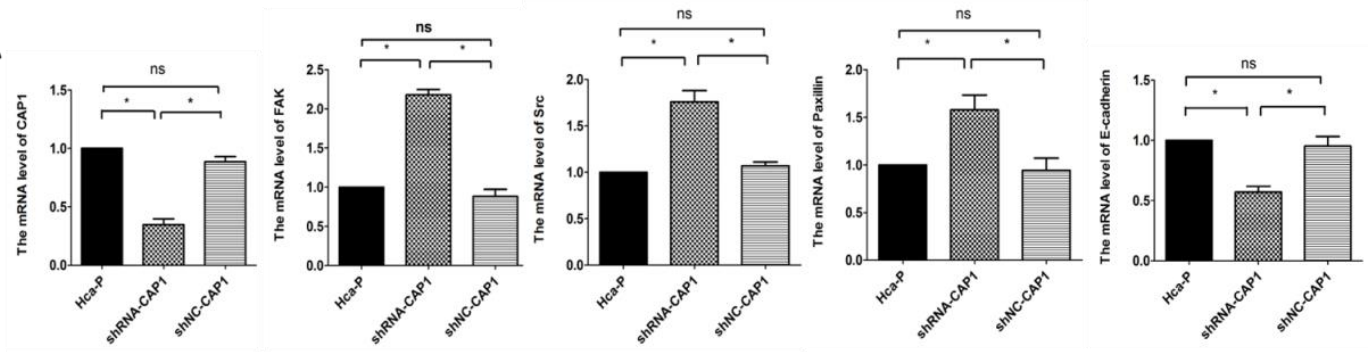

B
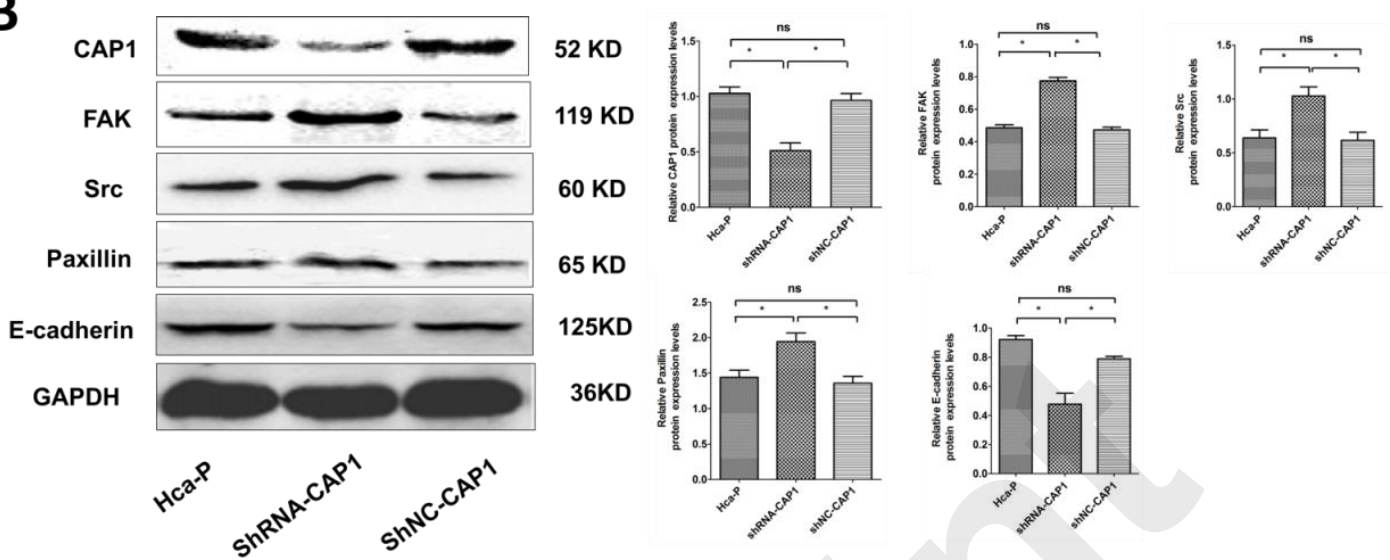

25KD
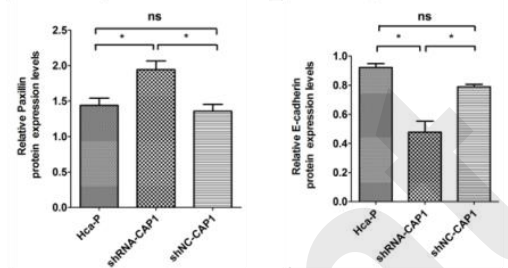

Fig. 3. (A) The mRNA expression levels of CAP1, FAK, Src, Paxillin and E-cadherin were detected by qRT-PCR in the three groups of cells. * indicates $\mathrm{P}<0.05$, ns represents $\mathrm{P}>0.05$. (B) On the left side of this figure, it shows the protein gel electrophoresis case of these three groups of cells; On the right side of this figure, the protein column comparison is demonstrated of the three groups of cells; * indicates $\mathrm{P}<0.05$, ns represents $\mathrm{P}>0.05$.

\subsection{Effects of CAP1 down-regulation on the biological behavior of Hca-p cells}

The biological behavior of cells such as growth, differentiation, proliferation, apoptosis, invasion, adhesion, migration, etc. can have a related impact on the occurrence and development of the tumor. We examined the effects of CAP1 down-regulation on the proliferation, apoptosis, invasion and adhesion abilities of Hca-P cells.

CCK-8 proliferation test was applied to detect the absorbance value of the ShRNA- CAP1, ShNC-CAP1 and Hca-P cell group setting at 0 h, 24 h, 48 h, 72 h, 96 $\mathrm{h}$ period. The absorbance values point mean standard deviation of three groups were shown in table 1. This experiment showed that CAP1 down-regulation lead the increased proliferation feature in Hca-P cells and suggested that CAP1 gene might inhibit the proliferation ability of the tumor cells (Fig.4 A). 


\begin{tabular}{cccccc}
\hline Time Nodes & $0 \mathrm{~h}$ & $24 \mathrm{~h}$ & $48 \mathrm{~h}$ & $72 \mathrm{~h}$ & $96 \mathrm{~h}$ \\
\hline ShRNA-CAP1 & $\begin{array}{c}0.106 \pm \\
0.015\end{array}$ & $0.152 \pm 0.010$ & $0.522 \pm 0.049$ & $0.651 \pm 0.040$ & $0.708 \pm 0.037$ \\
ShNC-CAP1 & $0.095 \pm$ & $0.144 \pm 0.024$ & $0.374 \pm 0.027$ & $0.485 \pm 0.018$ & $0.535 \pm 0.039$ \\
& 0.017 & & & & \\
Hca-P & $0.101 \pm$ & $0.153 \pm 0.006$ & $0.410 \pm 0.021$ & $0.553 \pm 0.022$ & $0.601 \pm 0.030$ \\
& 0.014 & & & $<0.05$ & $<0.05$ \\
P1 & $>0.05$ & $>0.05$ & $<0.05$ & $<0.05$ & $<0.05$ \\
P2 & $>0.05$ & $>0.05$ & $<0.05$ & $<0.05$ & $>0.05$ \\
P3 & $>0.05$ & $>0.05$ & $>0.05$ & $>0.05$ & \\
\hline
\end{tabular}

Table 1: The absorbance values point mean standard deviation of three groups

*P1 stands for comparison between group ShRNA-CAP1 and group ShNC-CAP1; P2 stands for comparison between group ShRNA-CAP1 and group Hca-P;P3 stands for comparison between group ShNC-CAP1 and group Hca-P.

Flow cytometry (FCM) was applied to detect whether CAP1 down-regulation could affect Hca-P cell early apoptosis function. The results showed that the early apoptosis cells of the three groups were $6.33 \% \pm 0.17 \%, 3.41 \% \pm 0.22 \%$ and $6.43 \% \pm$ $0.25 \%$. The early apoptotic ability of ShRNA-CAP1 cell group was lower than ShNC-CAP1 and Hca-P cell groups after lowering the gene expression of CAP1 (P < 0.05). We found that CAP1 down-regulation could inhibit the early apoptosis of Hca-P cells. (Fig.4 B, C).

Lymph node adhesion experiment was applied to inspect the influence of CAP1 down-regulation on cell adhesion ability. The adhesion cells number of each lymph 
node were $255.67 \pm 28.43 、 734.33 \pm 67.50 、 228.33 \pm 20.03$ of the three cell groups. It showed that the adhesion ability of ShRNA-CAP1 cell group was stronger than the ShNC-CAP1 and Hca-P cell groups $(\mathrm{P}<0.05)$. We surmised CAP1 gene might affect the lymph node adhesion of the tumor cells (Fig.4 D, E).

Transwell chambers cell invasion experiment was used to detect the migration of the cells. It showed that the cell numbers through the chambers were $61.00 \pm 8.51$ 、 $173.00 \pm 18.03 、 58.00 \pm 6.40$ of ShRNA-CAP1 cell, Hca-P cell and ShNC-CAP1 groups $(\mathrm{P}<0.05)$, Which indicated CAP1 gene had an effect on the invasion ability of different Hca-P cells and he invasion ability was increased with down-regulating CAP1(Fig.4 F,G).

A

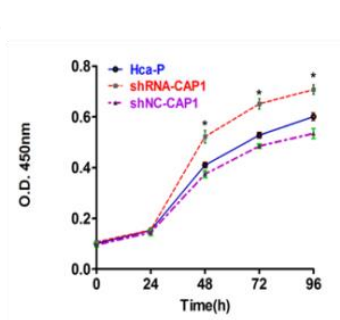

D
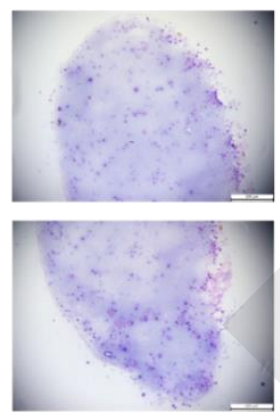

Hca-P

$\mathbf{F}$

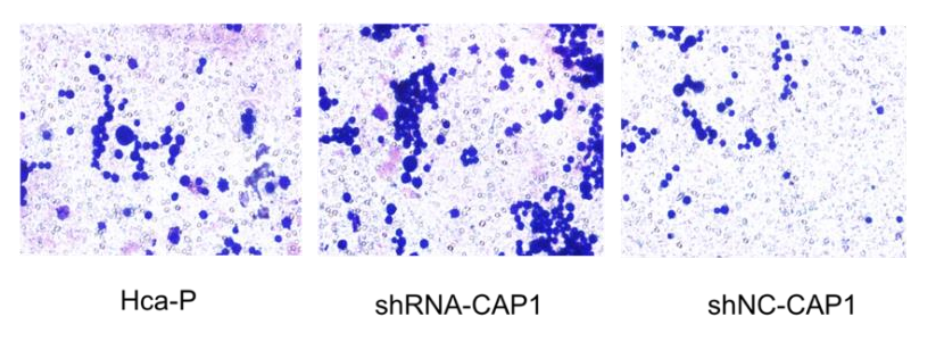

B
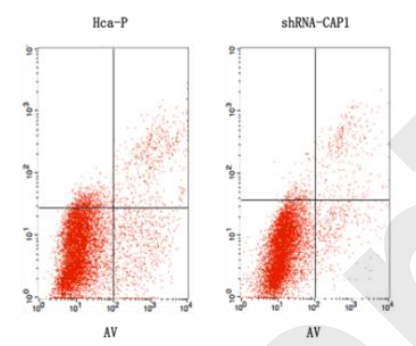

AV
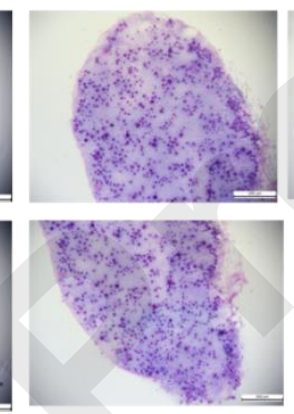

shRNA-CAP1

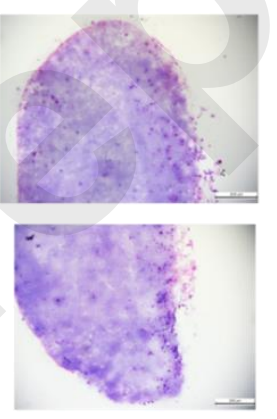

shNC-CAP1

shNC-CAP1
C

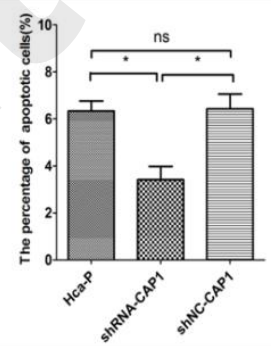

E

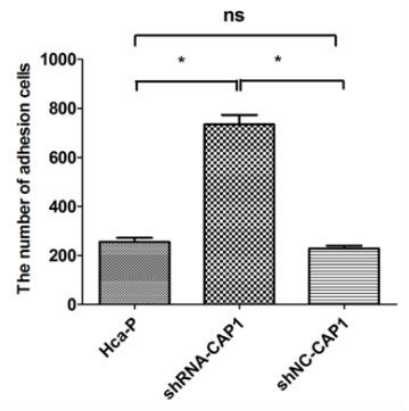

G

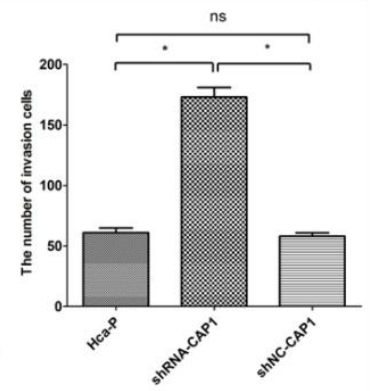

Fig. 4. (A)CCK-8 method was applied to detect the effect of CAP1 down-regulation on the proliferation of Hca-P cells. * P $<0.05$. (B)The effects of CAP1 down-regulation on early apoptosis by flow cytometry in different Hca-P cells. (C) This graph shows the apoptotic histograms of these three groups; * means $\mathrm{p}<0.05$. (D) The lymph node adhesion assay was used to detect 
the effect of CAP1 down-regulation on lymph node adhesion in Hca-P cells. The comparison of lymph node adhesion situation in three groups of cells is shown in this figure. (E)It shows the histogram of lymph node adhesion cells in the three groups of cells.* indicates $\mathrm{P}<0.05$, ns represents $\mathrm{P}>0.05$. (F)Transwell chamber method was applied to detect the effect of CAP1 gene expression on the invasion of Hca-P cells. The three groups of cells through the chambers were compared in this image. (G)It shows the histogram of invasion cells in the three groups of cells.* indicates $\mathrm{P}<0.05$, ns represents $\mathrm{P}>0.05$.

\subsection{The interaction between AnnexinA7 and CAP1.}

The analysis of the relationship between AnnexinA7 and CAP1 showed that after down-regulating the expression of AnnexinA7 gene, the level of gene and protein expression of ShRNA-CAP1 cell were $17.21 \%$ and $46.86 \%$ lower than ShNC-CAP1 group respectively $(\mathrm{P}<0.05)$. This indicated that low expression of AnnexinA7 gene caused the decline of CAP1 in gene and protein level. For further exploration, immunofluorescence was applied to find out the underlying relationship between AnnexinA7 and CAP1.We found that AnnexinA7 and CAP1 mainly located in the cytoplasm of the different group cells and a small amount of expression of them pitched in the nucleus. The image results reveal that the two molecules might position in cytoplasm of the approximation region (Fig.5 A, B, C, D). 
A

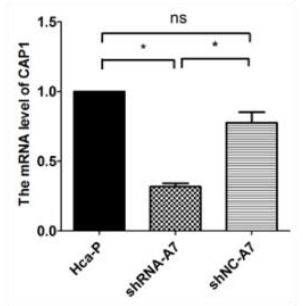

B

CAP1

GAPDH
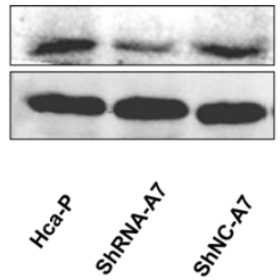

D

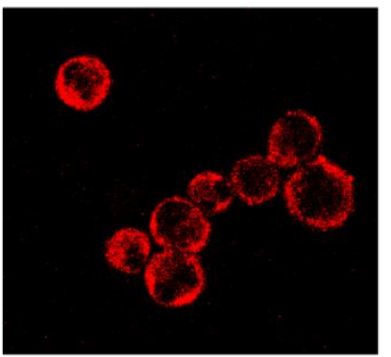

1

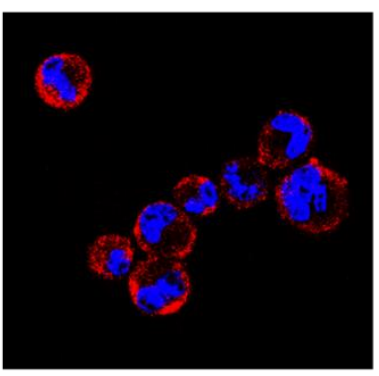

4

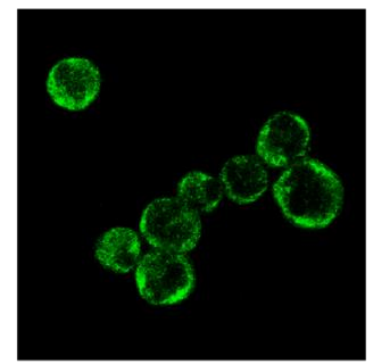

2

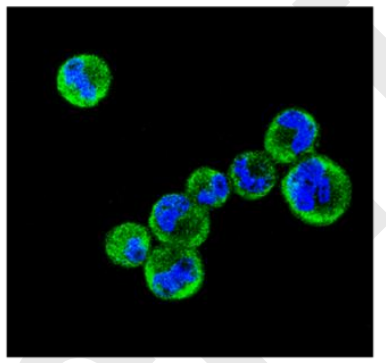

5
C
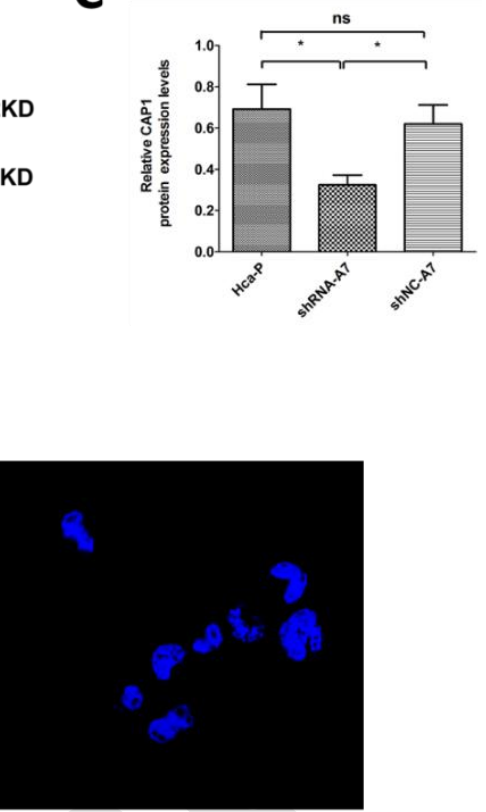

3

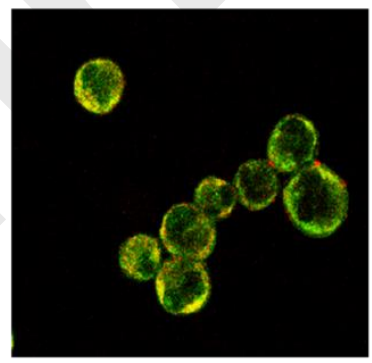

6

Fig. 5. (A)The effects of down-regulation of AnnexinA7 gene on CAP1 gene expression, *indicates $\mathrm{P}<0.05$, ns represents P> 0.05. (B) The effects of down-regulation of AnnexinA7 gene on CAP1 protein expression, this part showed the gel electrophoresis of CAP1 protein by influence of AnnexinA7 gene down - regulation. (C) The histogram of CAP1 protein expression level in the three groups. *indicates $\mathrm{P}<0.05$, ns means $\mathrm{P}>0.05$. (D)AnnexinA7 and CAP1 proteins were co-located in cells $(\times 400)$,D1:The location of AnnexinA7 in Hca-P cell;D2:The location of CAP1 in Hca-P cell;D3:The nucleus staining by DAPI; D4:AnnexinA7 overlaps with the nucleus;D5:CAP1 overlaps with the nucleus;D6:Annexin A7 overlaps with CAP1. 


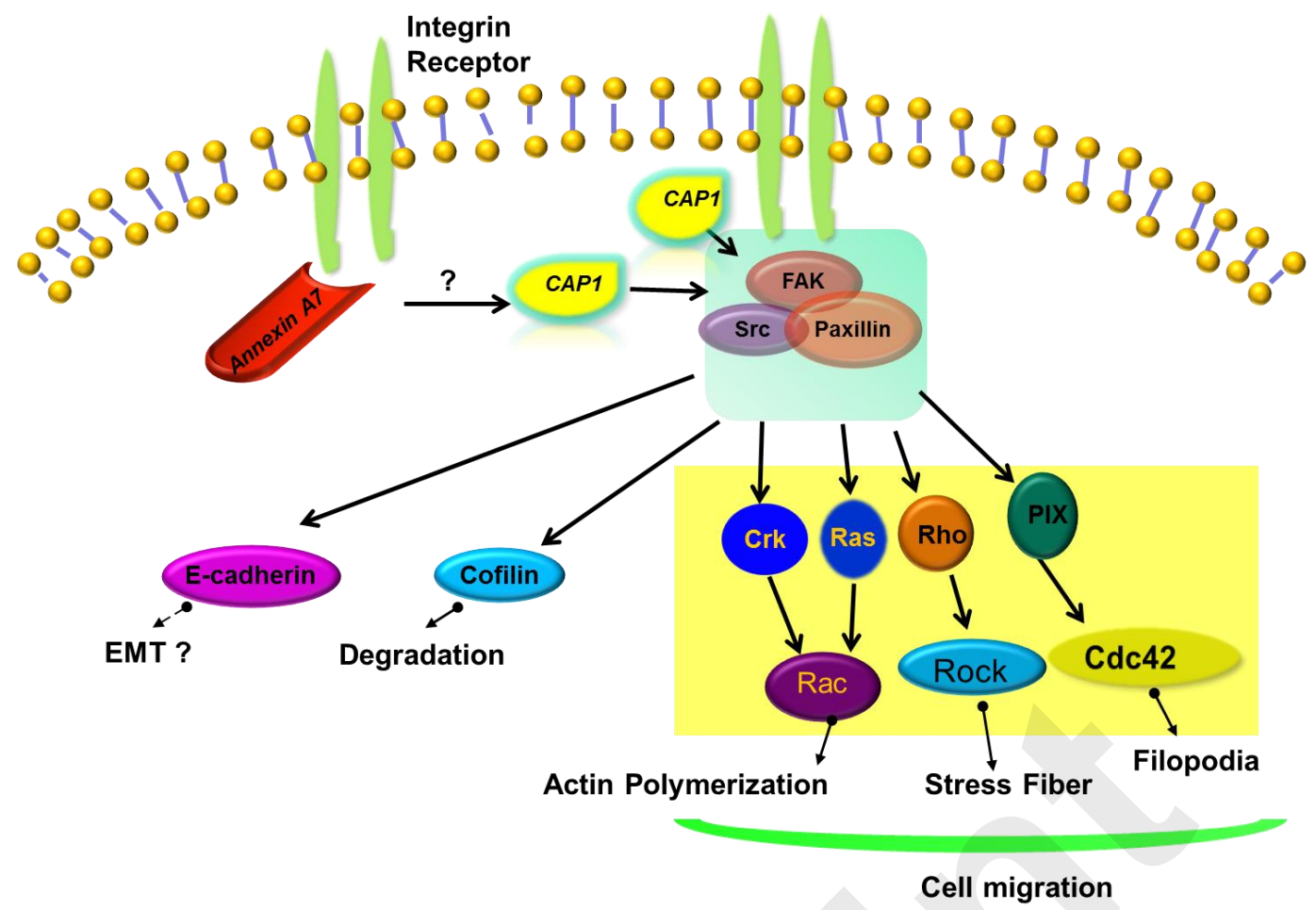

Fig. 6. The signal pathway relationship of the target molecules involved in this study,? indicates there may be an unclear association .

\section{Discussion}

Annexins is a family of $\mathrm{ca}^{2+}$ dependent phospholipid-binding proteins encoded by at least 12 genes, which is involved in cell signal transduction, inflammatory response, endocytosis and extracellular secretion, $\mathrm{ca}^{2+}$ ion channel formation, cytoskeletal protein interactions, tumor cell proliferation and differentiation, and maintenance of extracellular matrix integrity as well as many other aspects of cellular function [11]. We speculate Annexins may be a biomarker for further exploring the development and treatment of tumors through consulting previous research. AnnexinA7 plays various biological effects by activating the protein kinase $\mathrm{C}$ (PKC) and $\mathrm{ca}^{2+} /$ GTP signaling pathway as a membrane-related $\mathrm{ca}^{2+}$ regulatory protein. Moreover, AnnexinA7 is reported to be an important Ras pathway regulator and be involved in at least three signal pathways in cell cycle [12]. The low expression of AnnexinA7 can influence the expression of DNA repair genes, tumor suppressor genes and apoptosis-related genes by mediating the transduction pathways of discrete signaling in tumor cells, and thus cause the further development of tumor instability 
[13]. In multiple tumor cells, the low expression of AnnexinA7 is associated with the high invasion potential and its total protein distribution is related to the tumor suppressor effect, and it participates in neurotransmitters in homeostasis and carcinogenesis by regulating secretion/hormone secretion [14]. The upregulation of AnnexinA7 gene in Hca-P cells could enhance the lymphatic metastasis rate, while downregulation of AnnexinA7 in Hca-F cells decreased the rate of lymph node metastasis [1].

The adenylate cyclase synthase family (Cyclase-associated protein, CAP) is an important actin modulator consisting of 450-550 amino acids in eukaryotic organism. The rearrangement of cytoskeletal proteins plays a key role in cell migration. The CAP1 activation can affect cell activity by affecting actin kinetics [15]. Recent studies have shown that CAP1 deletion can stimulate cell motility through regulating the cell adhesion signaling pathway. The research about the relationship between CAP1 and adhesion molecules has found that CAP1 can form complex with FAK, activate the integrin signaling pathway molecules, and promote the local adhesion signal towards the downstream signal molecules, such as Src, Paxilin and actinin etal[16].And these adhesion molecules are important components of the extracellular matrix, which are not only involved in the presence of tumor microenvironment, but also participate in complex cell signal transduction.

For example, FAK is a kind of non-receptor tyrosine kinase, which is a key molecule of integrin signaling pathway. It is the core promoter of P53, AP-1, AP-2, NF-kB, PU-1, EGR-1 and TCF-1 and can participate in actin skeleton regulation, antigen processing and other transduction pathways. Above all, it is a key molecule in the process of lymph node metastasis because of regulating adhesion and migration abilities in tumor cell [17]. The FAK overexpression is an early biomarker of invasion and metastasis potential in different tumors [18,19,20]. The abnormal expression of FAK will lead the nest apoptosis lose, then increase cell DNA synthesis and accelerate G1 / S phase conversion [21]. Src is a member of SRC tyrosine kinase family, which can promote the growth of tumor cells and participate in cell adhesion, proliferation, apoptosis, differentiation and cell motility [22]. In the signal transduction pathway, Src can affect the molecule like FAK, PI3K, JNK, STAT3, Paxillin, MRK, ERK and p130cas, which are related to cell growth, migration and 
activation of angiogenic molecules [23]. In some reports on cell migration, the FAK/Src complex promotes the activation of FAK in combination with Src kinase substrates and plays an important role in actin activation and recombination. In addition, the inhibition of Src expression can increase the expression of E-cadherin protein, so the clinical inhibition of Src expression may improve the therapeutic effect [24-27]. The immunoprecipitation confirms that Src interacts with FAK under basic conditions, whereas FAK / Src complex may co-regulate with TGFb to the induction of thyroid cancer epithelial-mesenchymal transition (EMT). The E-cadherin expression can be inhibited by FAK / Src complex in tumor EMT pathway and can be involved in the maintenance of cell polarity, cell morphology and tissue integrity. At the same time, it is negatively correlated with the benign and malignant degree and the metastasis pathway of tumor cells as a tumor growth inhibitor [28]. In summary, the complex of FAK and Src regulates the expression of E-cadherin, which leads to the loss of polarity, the decrease of cell-to-cell junction and adhesion, and then increase the infiltration and migration ability, thereby enhancing the invasion and metastasis potential of tumor cells. As an another important regulator protein of the FAK / Src adhesion downstream, the low expression of Paxillin can affect the cell morphological changes and the formation of pseudopodia, which lead the ability of adhesion and migration decreased and the high expression of it cause the opposite result [29]. In tumor cells, Paxillin and FAK, Src could form the FAK-Src-Paxillin signal cascade, which can disrupt normal cell adhesion and cell proliferation, and involve changes in the cytoskeleton effect, forming important molecular mechanisms of tumor cell metastasis [30,31].

In this research, the degree of adhesion-related molecular gene or protein expression (FAK, Src, Paxillin and E-cadherin) is related to the down-regulation of the target molecules AnnexinA7 and CAP1. The expression trend of these adhesion-related molecular is consistent after AnnexinA7 and CAP1 down-regulation. For instance, the gene and protein levels of FAK, Src, Paxillin are increased in the shRNA-A7 and shRNA-CAP1 groups, but the E-cadherin expression level is decreased. Thus, we speculate that AnnexinA7 and CAP1 genes may have some relevance in molecular mechanism. The effects of AnnexinA7 and CAP1 on the expression of adhesion signaling molecules involved may be achieved by integrin 
protein in this study. AnnexinA7 can intervene in cell vesicle transport and membrane fusion through its GTPase activity and the conduction of discrete signaling. Existing data indicate that AnnexinA7 can regulate the phosphorylation and nuclear ectopic of integrin $\beta 4$ (ITGB4) molecules under the influence of certain factors, such as the changes of its GTPase activity. Moreover, it was found that AnnexinA7 could interact with integrin $\beta 4$ under immunoprecipitation and AnnexinA7 down-regulation could increase the integrin $\beta 4$ protein expression under certain conditions significantly [32]. In addition, integrin proteins are closely related to FAK, for example, it was found integrin $\beta 4$ and FAK interaction exists in the molecular cascade signal in breast cancer. In the molecular cascade signal, integrin can activate FAK and downstream signaling network, thus promoting the cancer progression. Molecules like integrin $\beta 4$, FAK and Paxillin can be combined to form an adhesive complex to be the intercellular structure of the connection. The adhesion complexes of the above three molecules attach to the cell basement membrane, and it also plays a role in the downstream of signal transduction pathways then transduce the extracellular signal into the cell.

We found that the gene and protein expression levels of CAP1 are down-regulated with the AnnexinA7 down-regulation by further analysis of the relationship between AnnexinA7 and CAP1.And then we found the expression and location of these two proteins are basically consistent by immunofluorescence assay. AnnexinA7 is mainly located in cytoplasm, plasma membrane, and nucleus, and our experiment also have confirmed that CAP1 is also located in the cytoplasm which is almost the same with the specific region of AnnexinA7.In combination with the mechanism described above, we hypothesized that AnnexinA7 may play an important role in the expression of cell adhesion molecules, which may be an up stream gene of the CAP1. From the schematic model we drew, we guess that AnnexinA7 and CAP1 may activate the signal cascade molecules of FAK, Src and Paxillin in the integrin signaling pathway, and then affect the expression of downstream molecules such as E-cadherin, Rho and PIX, so as to interfere with Epithelial-to-mesenchymal transition(EMT), degradation, cell migration and other behaviors. According to the results of this experiment, AnnexinA7 could affect the expression of CAP1.So it was 
speculated that AnnexinA7 may be one upstream molecular of CAP1(Fig.6). Of course, it requires follow up experiments to confirm further.

Therefore, we speculate that the down-regulation of AnnexinA7 and CAP1 expressions in Hca-P cells may have an effect on the expression of integrin protein and then generate a positive effect on FAK-Src-Paxillin signaling cascade. The complexes formed by FAK and Src regulate the expression of E-cadherin, which leads to the cell depolarized, adhesion decreased, and the invasion and migration ability increased, thereby enhancing the invasion and metastasis of tumor cells. However, the above-mentioned mechanism is rarely researched in hepatocellular carcinoma and different in different types of tumor cells. Some studies showed that the deletion of CAP1 enhances the cell adhesion signaling pathway and affects the mechanism of adhesion molecules expression. The above conclusion may be consistent with the mentioned AnnexinA7-related molecular regulation mechanism in this paper previously. And the functional experimental results show that after downregulating CAP1 gene, the abilities of proliferation, invasion and lymph node adhesion are increased in Hca-P cells, while the proportion of early apoptotic cells decreased, which could be used as the auxiliary basis for the above theory.

However, this study has limitations as follow:(1) Due to the time reason, our research only performed a down-regulation of the double target genes (AnnexinA7, CAP1) in Hca-P cells without a related treatment for up-regulation. (2) Our study just chose one cell line(Hca-P) of our research group ,but we did not do related experiments in Hca-F cells. We will do more thorough experiments to make up for the above shortcomings in the future.

\section{Acknowledgements}

This work was supported by grant from the National Natural Science Foundation of China [No. 81702834;81860089], the Financial Department of Liaoning Province [No. 20121203] and the Educational Department of Liaoning Province [No. L20133456].

\section{Compliance with ethical standards}

\section{Conflict of interest}


The authors declare no conflict of interest.

\section{Ethical approval}

This article does not contain any studies with human participants that were performed by any of the authors. Animal experiments were carried out according to National Institutes of Health Guide for the care and use of laboratory animals. The experimental protocol was approved by the Ethics Committee of Experimental Animals of Dalian Medical University.

\section{References}

【1】 Jin Y, Wang S, Chen W, et al. Annexin A7 suppresses lymph node metastasis of hepatocarcinoma cells in a mouse model[J]. BMC Cancer, 2013, 13(1):522.

【2】Srivastava M, Bubendorf L, Nolan L, et al. ANX7 as a Bio-Marker in Prostate and Breast Cancer Progression[J]. Disease Markers, 2001, 17(2):115-120.

【3】Carlotta Z, Gathof B S , Picker S M , et al. Function, expression and localization of annexin A7 in platelets and red blood cells: Insights derived from an annexin A7 mutant mouse[J]. BMC Biochemistry, 2003, 4(1):1-11.

【4】 Caterino M , Ruoppolo M , Stefania Orrù, et al. Characterization of red cell membrane proteins as a function of red cell density:: Annexin VII in different forms of hereditary spherocytosis[J]. Febs Letters, 2006, 580(28-29):6527-6532.

【5】 Ono, S. The role of cyclase-associated protein in regulating actin filament dynamics - more than a monomer-sequestration factor[J]. Journal of Cell Science, 2013, 126(15):3249-3258.

【6】Agrawal P B , Greenleaf R S, Tomczak K K, et al. Nemaline Myopathy with Minicores Caused by Mutation of the CFL2 Gene Encoding the Skeletal Muscle Actin-Binding Protein, Cofilin-2[J]. American Journal of Human Genetics, 2007, 80(1):0-167.

【7】Zhang $\mathrm{H}$, Ghai $\mathrm{P}, \mathrm{Wu} \mathrm{H}$, et al. Mammalian Adenylyl Cyclase-associated Protein 1 (CAP1) Regulates Cofilin Function, the Actin Cytoskeleton, and Cell Adhesion[J]. Journal of Biological Chemistry, 2014, 288(1):20966-77.

【8】Fan Y C , Cui C C , Zhu Y S , et al. Overexpression of CAP1 and its significance in tumor cell proliferation, migration and invasion in glioma[J]. Oncology Reports, 2016. 
【9】 Li M , Yang X, Shi H, et al. Downregulated Expression of the Cyclase-associated Protein 1 (CAP1) Reduces Migration in Esophageal Squamous Cell Carcinoma[J]. Japanese Journal of Clinical Oncology, 2013, 43(9):856-864.

【10】Cun W , Kai J , Dongmei G, et al. Clusterin Protects Hepatocellular Carcinoma Cells from Endoplasmic Reticulum Stress Induced Apoptosis through GRP78[J]. PLoS ONE, 2013, 8(2):e55981-.

【11】 Srivastava, M. Prognostic Impact of ANX7-GTPase in Metastatic and HER2-Negative Breast Cancer Patients[J]. Clinical Cancer Research, 2004, 10(7):2344-2350.

【12】 Ji H , Moritz R L, Kim Y S , et al. Analysis of Ras-induced oncogenic transformation of NIH-3T3 cells using differential-display 2-DE proteomics[J]. Electrophoresis, 2007, 28(12):1997-2008.

【13】 Srivastava M , Montagna C, Leighton X , et al. Haploinsufficiency of Anx7 tumor suppressor gene and consequent genomic instability promotes tumorigenesis in the Anx7(+/-) mouse[J]. Proceedings of the National Academy of Sciences, 2003, 100(24):14287-14292.

【14】 Sidiropoulou T, Karaferi A, Stachtos G, et al. Propofol but not sevoflurane decreases circulating levels of sEGFR and sE-selectin after colorectal cancer surgery[J]. Archives of Medical Science - Civilization Diseases, 2019, 4(1):41-50.

【15】 Hubberstey A V , Mottillo E P . Cyclase-associated proteins: CAPacity for linking signal transduction and actin polymerization[J]. The FASEB Journal, 2002, 16(6):487-499.

【16】 Legate K R, Wickstrom S A, Fassler R . Genetic and cell biological analysis of integrin outside-in signaling[J]. Genes \& Development, 2009, 23(4):397-418.

【17】 Hecker T P, Gladson C L. Focal adhesion kinase in cancer[J]. Frontiers in Bioscience A Journal \& Virtual Library, 2003, 8(1-3):s705-14.

【18】 Miyazaki T , Kato H , Nakajima M , et al. FAK overexpression is correlated with tumour invasiveness and lymph node metastasis in oesophageal squamous cell carcinoma[J]. British Journal of Cancer, 2003, 89(1):140-145.

【19】 Kondo $\mathrm{Y}$. Treatment of prostate cancer in vitro and in vivo with 2-5A-anti-telomerase RNA component[J]. Oncogene, 2000, 19. 
【20】Zhao J , Pestell R , Guan J L . Transcriptional Activation of Cyclin D1 Promoter by FAK Contributes to Cell Cycle Progression[J]. Molecular Biology of the Cell, 2001, 12(12):4066-4077.

【21】Kwong L , Wozniak M A , Collins A S , et al. R-Ras Promotes Focal Adhesion Formation through Focal Adhesion Kinase and p130Cas by a Novel Mechanism That Differs from Integrins[J]. Molecular and Cellular Biology, 2003, 23(3):933-949.

【22】Zheng R , Qin X , Wenjie L I . Effect of Src Tyrosine Kinase Inhibition on Secretion of MMP-2 and MMP-9 by Non-small Cell Lung Cancer Cells[J]. Chinese Journal of Lung Cancer, 2011.

【23】 Wheeler D L, Iida M , Dunn E F . The Role of Src in Solid Tumors[J]. The Oncologist, 2009, 14(7):667-678.

【24】Zhang J , Kalyankrishna S , Wislez M, et al. Src-Family Kinases Are Activated in Non-Small Cell Lung Cancer and Promote the Survival of Epidermal Growth Factor Receptor-Dependent Cell Lines[J]. American Journal of Pathology, 2007, 170(1):0-376.

【25】 Wilson G R , Cramer A , Welman A, et al. Activated c-SRC in ductal carcinoma in situ correlates with high tumour grade, high proliferation and HER2 positivity[J]. British Journal of Cancer, 2006, 95(10):1410-1414.

【26】 Song, L. Dasatinib (BMS-354825) Selectively Induces Apoptosis in Lung Cancer Cells Dependent on Epidermal Growth Factor Receptor Signaling for Survival[J]. Cancer Research, 2006, 66(11):5542-5548.

【27】 Cheng S Y S , Sun G, Schlaepfer D D, et al. Grb2 Promotes Integrin-Induced Focal Adhesion Kinase (FAK) Autophosphorylation and Directs the Phosphorylation of Protein Tyrosine Phosphatase? by the Src-FAK Kinase Complex[J]. Molecular and Cellular Biology, 2014, 34(3):348-361.

【28】 Du T , Qu Y , Li J , et al. Maternal embryonic leucine zipper kinase enhances gastric cancer progression via the FAK/Paxillin pathway[J]. Molecular Cancer, 2014, 13(1):100.

【29】Hagel M, George E L, Kim A, et al. The Adaptor Protein Paxillin Is Essential for Normal Development in the Mouse and Is a Critical Transducer of Fibronectin Signaling[J]. Molecular and Cellular Biology, 2002, 22(3):901-915. 
【30】 Xia P, Li S, Zhao Z, et al. Expression and clinical significance of FAK,Paxillin and MMP-9 protein in esophageal squamous cell carcinoma tissue[J]. Chongqing Medicine, 2011.

【31】 Sit S T , Manser E . Rho GTPases and their role in organizing the actin cytoskeleton[J]. Journal of Cell Science, 2011, 124(5):679-683.

【32】 Li H , Huang S, Wang S, et al. Relationship between annexin A7 and integrin $\beta 4$ in autophagy[J]. The International Journal of Biochemistry \& Cell Biology, 2013, 45(11):2605-2611. 


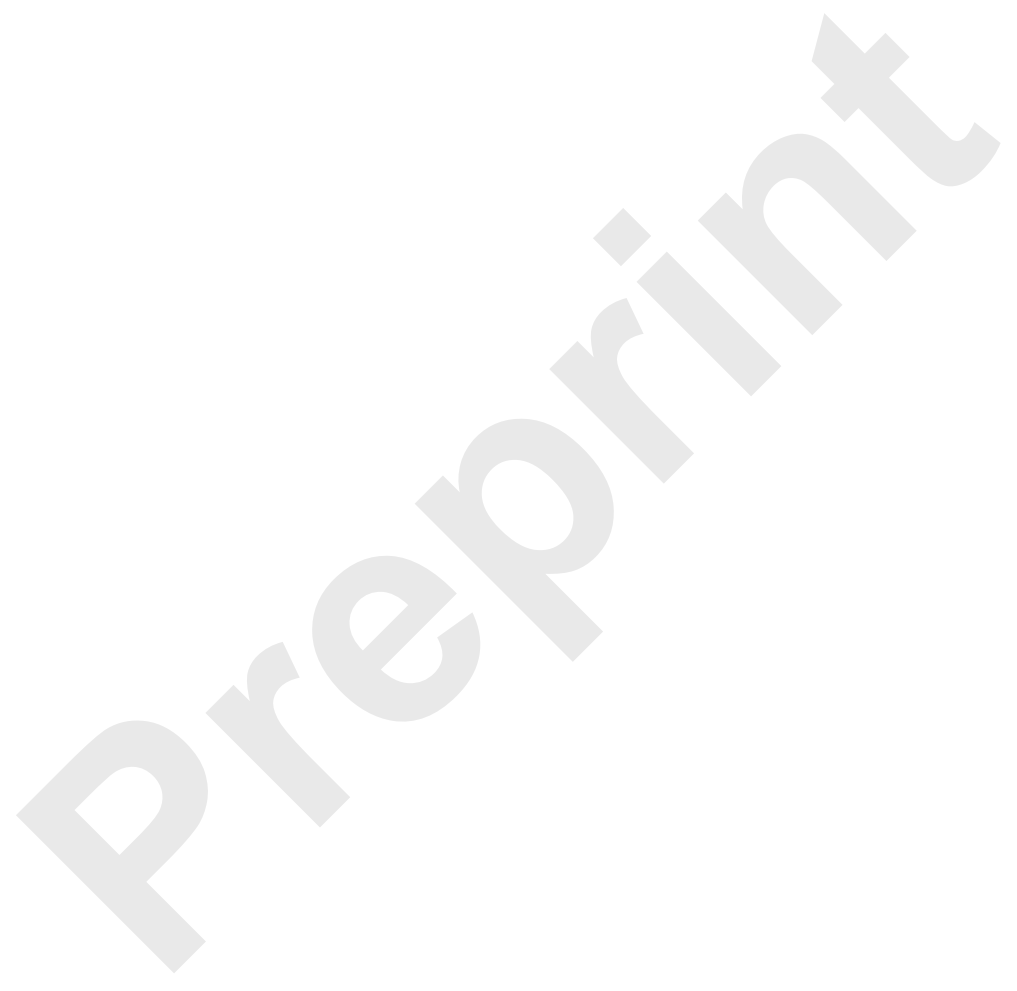

